\title{
Reductive Ring Opening of 3,5-Bis(2-arylethenyl)isoxazoles with Molybdenum Hexacarbonyl: A Novel Route to Symmetrical and Unsymmetrical Curcumin Derivatives
}

\author{
VIWAT HAHNVAJANAWONG*, THANAPHAT THAIMA, \\ RUCHANOK TEARAVARICH and PARINYA THERAMONGKOL
}

\author{
Department of Chemistry and Center for Innovation in Chemistry, Faculty of Science, \\ Khon Kaen University, Khon Kaen 40002, Thailand. \\ ${ }^{*}$ Corresponding author E-mail: viwhah@kku.ac.th \\ http://dx.doi.org/10.13005/ojc/320113
}

(Received: December 04, 2015; Accepted: February 02, 2016)

\begin{abstract}
Curcumin derivatives were successfully synthesized from 3,5-dimethylisoxazole by lateral metalation and condensation with various aromatic aldehydes sequentially at $\mathrm{C}_{5}-$ and $\mathrm{C}_{3}$-methyl groups. After dehydration, further transformation of isoxazole ring to $\beta$-diketone moiety was accomplished by reductive ring opening using molybdenum hexacarbonyl $\left[\mathrm{Mo}(\mathrm{CO})_{6}\right]$ and subsequent simple acidic hydrolysis.
\end{abstract}

Key words : Curcumin derivatives, Reductive ring opening, Molybdenum hexacarbonyl, Lateral metalation

\section{INTRODUCTION}

Curcumin, 1,7-bis(4-hydroxy-3methoxyphenyl)-1,6-heptadien-3,5-dione, is a bioactive compound isolated from Curcuma longa rhizomes. Curcumin has been reported to possess antioxidant, anti-inflammatory, antitmicrobial and anticarcinogenic activities. ${ }^{1}$ Various curcumin derivatives have been synthesized for testing their biological activities. Synthetic symmetrical curcumin derivatives reported in literature ${ }^{2-5}$ were obtained from Pabons procedure. ${ }^{6}$ Unsymmetrical curcumin derivatives, on the other hand, were synthesized by solid-phase synthetic strategy. ${ }^{7}$ Both syntheses proceeded through acetylacetone-boric anhydride complex which methyl terminals of this complex were allowed to undergo aldol condensation.

Isoxazoles can be employed as masked 1,3-dicarbonyl compounds because reductive cleavage of the $\mathrm{N}-\mathrm{O}$ bond in isoxazoles produce $\beta$-aminoenones which are readily transform to their corresponding $\beta$-hydroxyenones. We have envisioned for using this $\mathrm{N}-\mathrm{O}$ bond cleavage in our strategy for the synthesis of symmetrical as well as a wide range of unsymmetrical curcumin derivatives 
1, i.e., using isoxazole rings of 3,5-bis(2-arylethenyl) isoxazoles 2 as masked 1,3-dicarbonyl moieties of curcumin derivatives 1 (Scheme 1). The synthetic direction to obtain 2 involves transformation of methyl groups of 3,5-dimethylisoxazole (5) into 2-aryl-2hydroxyethyl groups sequentially at $\mathrm{C}_{5}$ and $\mathrm{C}_{3}$ to get 5-(2-aryl-2-hydroxyethyl)-3-methylisoxazoles 4 and 3,5-bis(2-aryl-2-hydroxyethyl)isoxazoles 3 respectively. Subsequent dehydration of 3,5-bis(2aryl-2-hydroxyethyl)isoxazoles 3 provides the route to 3,5-bisstyrylisoxazoles 2.

\section{Experimental Section General}

Melting points were determined by usinga Sanyo Gallenkamp melting point apparatus and are uncorrected. IR spectra were taken with a Perkin Elmer Spectrum One FT-IR Spectrometer. ${ }^{1} \mathrm{H}$ and ${ }^{13} \mathrm{C}$ NMR spectra were recorded using a VARIAN MERCURY plus (400 MHz FT NMR).

$n$-BuLi and $s$-BuLi were prepared and their concentration were determined following the standard procedure. ${ }^{8,9}$

\section{Preparation of 3,5-dimethylisoxazole (5) ${ }^{10}$}

A solution of hydroxylamine hydrochloride $(8.34 \mathrm{~g}, 0.12 \mathrm{~mole})$ in water $(10 \mathrm{ml})$ was added a solution of 2,4-pentanedione (6) (10.2 g, $0.1 \mathrm{~mole}$ ) in ethanol $(10 \mathrm{ml})$. The mixture was heated under reflux temperature for 3 hours, then allowed to cool to room temperature and poured into cold water $(60 \mathrm{ml})$. The resulting mixture was extracted with ether $(3 \times 40 \mathrm{ml})$. The combined organic layer was dried $\left(\mathrm{Na}_{2} \mathrm{SO}_{4}\right)$ and evaporated to dryness under reduced pressure. The brown oily residue was purified by distilling under reduce pressure to give 3,5-dimethylisoxazole (5) $\left(8.10 \mathrm{~g}, 82 \%\right.$ ) as a colourless liquid (bp $85-86^{\circ} \mathrm{C}$ at $150 \mathrm{mmHg}$ ); IR (thin film) $v_{\max }$ : $3132,2934,1611$, 1455, 1414, 1258, 1011, 886, $795 \mathrm{~cm}^{-1} ;{ }^{1} \mathrm{H}$ NMR $\left(\mathrm{CDCl}_{3}, 400 \mathrm{MHz}\right) \delta: 5.82(1 \mathrm{H}, \mathrm{s}, \mathrm{H}-4), 2.36(3 \mathrm{H}, \mathrm{s}$, $\left.\mathrm{C}=\mathrm{CCH}_{3}\right), 2.23\left(3 \mathrm{H}, \mathrm{s}, \mathrm{N}=\mathrm{CCH}_{3}\right) ;{ }^{13} \mathrm{C} \mathrm{NMR}\left(\mathrm{CDCl}_{3}\right)$ $\delta: 11.3,12.1,102.3,159.9,169.1$.

General procedure for preparation of 5-(2-aryl-2hydroxyethyl)-3-methylisoxazoles 4a-c

To a stirred solution of 3,5-dimethylisoxazole (5) $(2.91 \mathrm{~g}, 30.0 \mathrm{mmol})$ in dry THF $(60 \mathrm{ml})$ was added $n$-BuLi (1.0 M in hexane; $30 \mathrm{ml}, 30.0 \mathrm{mmol}$ ) dropwise under $\mathrm{N}_{2}$ at $-78{ }^{\circ} \mathrm{C}$ and the mixture was stirred for an additional1 hour. A solution of aromatic aldehyde $(30.0 \mathrm{mmol})$ in THF $(6 \mathrm{ml})$ was then added. The resulting mixture was allowed to warm up to room temperature and treated with water. The phases were separated and the aqueous layer was extracted with ethyl acetate $(3 \times 120 \mathrm{ml})$. The combined organic layer was dried $\left(\mathrm{Na}_{2} \mathrm{SO}_{4}\right)$ and concentrated under reduce pressure. Purification of the residue using column chromatography on silica gel with a gradient of 10 $50 \%$ ethyl acetate in hexane as eluent gave 5-(2-aryl2-hydroxyethyl)-3-methylisoxazoles $4 a-c$ (70-84\%) as yellow oils or as white crystalline solids.

\section{5-(2-Phenyl-2-hydroxyethyl)-3-methylisoxazole} (4a)

Yield: $4.69 \mathrm{~g}(77 \%)$; yellow oil; IR (thin film) $v_{\max }: 3370,3031,2931,1604,1493,1450$, $1415,1051,742,700 \mathrm{~cm}^{-1} ;{ }^{1} \mathrm{H} \mathrm{NMR}\left(\mathrm{CDCl}_{3}, 400 \mathrm{MHz}\right)$

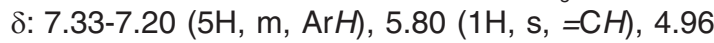
$(1 \mathrm{H}, \mathrm{dd}, J=8.2$ and $5.1 \mathrm{~Hz}, \mathrm{OCH}), 3.10(1 \mathrm{H}, \mathrm{dd}, J$ $=15.2$ and $8.2 \mathrm{~Hz}, \mathrm{HCH}), 3.01(1 \mathrm{H}, \mathrm{dd}, J=15.2$ and $5.0 \mathrm{~Hz}, \mathrm{HCH}), 2.12\left(3 \mathrm{H}, \mathrm{s}, \mathrm{CH}_{3}\right) ;{ }^{13} \mathrm{C} \mathrm{NMR}\left(\mathrm{CDCl}_{3}\right) \delta$ : 11.3, 36.6, 72.1, 103.4, 125.7, 128.0, 128.6, 143.0, 159.8, 169.6 .

[2-(4-Methoxyphenyl)-2-hydroxyethyl]-3methylisoxazole (4b)

Yield: $4.90 \mathrm{~g}(70 \%)$; yellow oil; IR (thin film) $v_{\text {max }}: 3376,2934,2838,1608,1513,1416,1246$, $1177,1032,833 \mathrm{~cm}^{-1} ;{ }^{1} \mathrm{H}$ NMR $\left(\mathrm{CDCl}_{3}, 400 \mathrm{MHz}\right) \delta$ : $7.21(2 \mathrm{H}, \mathrm{d}, J=8.7 \mathrm{~Hz}, \mathrm{ArH}), 6.82(2 \mathrm{H}, \mathrm{d}, J=8.6 \mathrm{~Hz}$, $\mathrm{Ar} H), 5.80(1 \mathrm{H}, \mathrm{s},=\mathrm{CH}), 4.92(1 \mathrm{H}, \mathrm{dd}, J=8.0$ and $5.4 \mathrm{~Hz}, \mathrm{OCH}), 3.74\left(3 \mathrm{H}, \mathrm{s}, \mathrm{OCH}_{3}\right), 3.10(1 \mathrm{H}, \mathrm{dd}, J=$ 15.2 and $8.1 \mathrm{~Hz}, \mathrm{HCH}), 2.99(1 \mathrm{H}, \mathrm{dd}, J=15.2$ and $5.3 \mathrm{~Hz}, \mathrm{HCH}), 2.15\left(3 \mathrm{H}, \mathrm{s}, \mathrm{CH}_{3}\right) ;{ }^{13} \mathrm{C} \mathrm{NMR}\left(\mathrm{CDCl}_{3}\right)$ $\delta: 11.2,36.5,55.2,71.4,103.2,113.8,127.0,135.4$, $159.1,159.7,169.9$.

[2-(3,4-Dimethoxyphenyl)-2-hydroxyethyl]-3methylisoxazole (4c)

Yield: $6.63 \mathrm{~g}(84 \%)$; white crystals; $\mathrm{mp}$ 63$64{ }^{\circ} \mathrm{C}$; IR (thin film) $v_{\max }: 3382,2936,1603,1513$, 1416, 1260, 1233, 1137, 1023, 809, $763 \mathrm{~cm}^{-1} ;{ }^{1} \mathrm{H}$ $\operatorname{NMR}\left(\mathrm{CDCl}_{3}, 400 \mathrm{MHz}\right) \delta: 6.90-6.78(3 \mathrm{H}, \mathrm{m}, \mathrm{ArH})$, $5.83(1 \mathrm{H}, \mathrm{s},=\mathrm{CH}), 4.98(1 \mathrm{H}, \mathrm{m}, \mathrm{OCH}), 3.83(6 \mathrm{H}, \mathrm{s}$, $\left.2 \times \mathrm{OCH}_{3}\right), 3.14(1 \mathrm{H}, \mathrm{dd}, J=15.1$ and $8.2 \mathrm{~Hz}, \mathrm{HCH})$, $3.04(1 \mathrm{H}, \mathrm{dd}, J=15.1$ and $5.1 \mathrm{~Hz}, \mathrm{HCH}), 2.20(3 \mathrm{H}$, $\left.\mathrm{s}, \mathrm{CH}_{3}\right) ;{ }^{13} \mathrm{C} \mathrm{NMR}\left(\mathrm{CDCl}_{3}\right) \delta: 11.3,36.7,55.9,71.7$, 103.5, 109.1, 111.2, 118.1, 136.1, 148.6149 .1 , $160.0,170.0$. 
General procedure for preparation of 3,5-bis(2aryl-2-hydroxyethyl)isoxazoles 3a-f

To a solution of 5-(2-aryl-2-hydroxyethyl)3-methylisoxazole $(4.0 \mathrm{mmol})$ in THF $(20 \mathrm{ml})$ was added $s$-BuLi (1.0 $\mathrm{M}$ in hexane, $10.0 \mathrm{ml}, 10.0 \mathrm{mmol}$ ) dropwise under $\mathrm{N}_{2}$ at $-78{ }^{\circ} \mathrm{C}$. After being stirred at $-78^{\circ} \mathrm{C}$ for 1 hour, a solution of aromatic aldehyde $(5.0 \mathrm{mmol})$ in THF $(2 \mathrm{ml})$ was then added. The reaction mixture was allowed to warm up to room temperature and treated with water. The phases were separated and the aqueous layer was extracted with ethyl acetate $(3 \times 50 \mathrm{ml})$. The combined organic layer was dried $\left(\mathrm{Na}_{2} \mathrm{SO}_{4}\right)$ and concentrated under reduced pressure. Purification of the residue using column chromatography on silica gel with agradient of $30-70 \%$ ethyl acetate in hexane as an eluent gave3,5-bis(2-aryl-2-hydroxyethyl)isoxazoles 3a-f (46-65\%) as crystalline solids or as oils.

\section{3,5-bis(2-Phenyl-2-hydroxyethyl)isoxazole (3a)}

Yield: $0.59 \mathrm{~g} \mathrm{(48 \% );} \mathrm{white} \mathrm{crystals;} \mathrm{mp} 97$ $98^{\circ} \mathrm{C}$; IR (thin film) $v_{\max }: 3377,3031,1601,1428$, 1052, 754, $700 \mathrm{~cm}^{-1} ;{ }^{1} \mathrm{H}$ NMR $\left(\mathrm{CDCl}_{3}, 400 \mathrm{MHz}\right)$ $\delta: 7.38-7.16(10 \mathrm{H}, \mathrm{m}, \mathrm{ArH}), 5.89$ and $5.88(1 \mathrm{H}, 2 \mathrm{~s}$, $=\mathrm{CH}), 5.03-4.84\left(2 \mathrm{H}, \mathrm{m}, 2^{\prime \prime}\right.$-and2'-OCH), $3.09(1 \mathrm{H}$, $\mathrm{dd}, J=15.2$ and $8.7 \mathrm{~Hz}, 1 "-H C H), 3.02(1 \mathrm{H}, \mathrm{dd}, J=$ 15.2 and 4.6 Hz, 1"-HCH), 2.98-2.89 (2H, m, 1'-CH $)$; ${ }^{13} \mathrm{C} \mathrm{NMR}\left(\mathrm{CDCl}_{3}\right)$ : $: 36.0,36.7,71.9,72.4,103.3$, $125.7,125.8,127.8,128.0$, 128.5, 128.6 , 142.9, 143.2, 161.4, 169.9 .

\section{3,5-Bis(2-(4-methoxyphenyl)-2-hydroxyethyl)} isoxazole (3b)

Yield: $0.72 \mathrm{~g}$ (49\%); white crystals; mp 97$99^{\circ} \mathrm{C}$; IR (thin film) $v_{\text {max }}: 3377,2957,1608,1514$, 1253, 1175, 1033, $825 \mathrm{~cm}^{-1} ;{ }^{1} \mathrm{H}$ NMR $\left(\mathrm{CDCl}_{3}, 400\right.$ $\mathrm{MHz}) \delta: 7.27(4 \mathrm{H}, \mathrm{d}, J=8.4 \mathrm{~Hz}, \operatorname{ArH}), 6.88(4 \mathrm{H}, \mathrm{d}, J=$ $8.4 \mathrm{~Hz}, \mathrm{ArH}) 5.88$ and $5.87(1 \mathrm{H}, 2 \mathrm{~s},=\mathrm{CH}), 5.03-4.91$ $\left(2 \mathrm{H}, \mathrm{m}, 2^{\prime \prime}-\right.$ and 2'-OCH), $3.80\left(6 \mathrm{H}, \mathrm{s}, 2 \mathrm{xOCH}_{3}\right), 3.16$ $(1 \mathrm{H}, \mathrm{dd}, J=15.2$ and $8.4 \mathrm{~Hz}, 1 "-\mathrm{HCH}), 3.10-2.92(3 \mathrm{H}$,

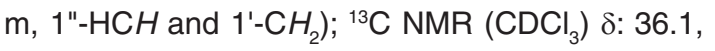

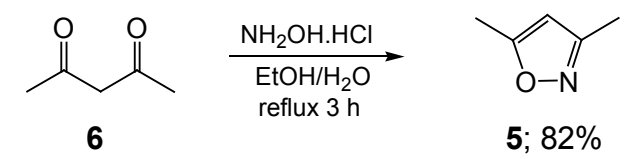

36.6, 55.3, 71.7, 72.1, 103.3, 113.9, 114.0, 127.0, $127.1,135.1,135.4,159.2,159.4,161.4,169.9$.

\section{3,5-Bis[2-(3,4-dimethoxyphenyl)-2-hydroxyethyl] isoxazole (3c)}

Yield: $1.12 \mathrm{~g}(65 \%)$; yellow crystals; $\mathrm{mp}$ 89-91 ${ }^{\circ} \mathrm{C}$; IR (thin film) $v_{\max }: 3384,2937,1599,1513$, 1420, 1260, 1138, 1022, 811, $762 \mathrm{~cm}^{-1} ;{ }^{1} \mathrm{H}$ NMR $\left(\mathrm{CDCl}_{3}, 400 \mathrm{MHz}\right) \delta: 6.89(1 \mathrm{H}, \mathrm{s}, \mathrm{ArH}), 6.85(1 \mathrm{H}, \mathrm{s}$, $\mathrm{ArH})$, 6.84-6.74 (4H, m, ArH), 5.90 and $5.87(1 \mathrm{H}$, $2 \mathrm{~s},=\mathrm{CH}), 4.93-4.81\left(2 \mathrm{H}, \mathrm{m}, 2^{\prime \prime}-\right.$ and $\left.2^{\prime}-\mathrm{OCH}\right), 3.81$ and $3.80\left(12 \mathrm{H}, 2 \mathrm{~s}, 4 \times \mathrm{OCH}_{3}\right), 3.08(1 \mathrm{H}, \mathrm{d} \mathrm{d}, J=15.2$ and $8.7 \mathrm{~Hz}, 1$ "- $\mathrm{HCH}), 3.03-2.86(3 \mathrm{H}, \mathrm{m}, 1$ "-HCH and $\left.1^{\prime}-\mathrm{CH}_{2}\right) ;{ }^{13} \mathrm{C}$ NMR $\left(\mathrm{CDCl}_{3}\right) \delta: 36.0,36.8,55.8,55.9$, 71.7, 72.3, 103.3, 108.9, 109.1, 111.0, 117.9, 118.0, 135.8, 136.0, 148.4, 148.5, 148.9, 161.4, 169.9.

\section{3 - [2 - P he n y I- 2- h y d rox y e th y I]- 5 - ( 2 - ( 4 - methoxyphenyl)-2-hydroxyethyl)isoxazole (3d)}

Yield: $0.77 \mathrm{~g}$ (57\%); brown crystals; mp 63-64 ${ }^{\circ} \mathrm{C}$; IR (thin film) $v_{\max }: 3365,2896,1606,1513$, 1437, 1246, 1176, 833, $701 \mathrm{~cm}^{-1}$; ${ }^{1} \mathrm{H} \mathrm{NMR}\left(\mathrm{CDCl}_{3}\right.$, $400 \mathrm{MHz}) \delta: 7.34-7.20(5 \mathrm{H}, \mathrm{m}, \mathrm{ArH}), 7.16(2 \mathrm{H}, \mathrm{d}, \mathrm{J}$ $=8.7 \mathrm{~Hz}, \operatorname{ArH}), 6.80(2 \mathrm{H}, \mathrm{d}, J=8.7 \mathrm{~Hz}, \mathrm{ArH}), 5.85$ and $5.84(1 \mathrm{H}, 2 \mathrm{~s},=\mathrm{CH}), 4.91-4.80(2 \mathrm{H}, \mathrm{m}, 2$ "-and 2'-OCH), $3.72\left(3 \mathrm{H}, \mathrm{s}, \mathrm{OCH}_{3}\right), 3.03(1 \mathrm{H}, \mathrm{dd}, J=15.2$ and $8.6 \mathrm{~Hz}, 1 "-\mathrm{HCH}), 2.99-2.83(3 \mathrm{H}, \mathrm{m}, 1 "-\mathrm{HCH}$ and $\left.1^{\prime}-\mathrm{CH}_{2}\right) ;{ }^{13} \mathrm{C}$ NMR $\left(\mathrm{CDCl}_{3}\right) \delta: 36.2,36.6,55.3$, $71.8,72.5,103.3,114.0,125.8,127.0,127.8,128.5$, 135.0, 143.2, 159.4, 161.3, 169.9 .

\section{3-[2-Phenyl-2-hydroxyethyl]-5-(2-(3,4- dimethoxyphenyl)-2-hydroxyethyl) isoxazole (3 e)}

Yield: $0.75 \mathrm{~g}$ (51\%); yellow oil; IR (thin film) $v_{\max }: 3381,2938,1600,1514,1421,1260,1138$, $1022,810,760,701 \mathrm{~cm}^{-1} ;{ }^{1} \mathrm{H}$ NMR $\left(\mathrm{CDCl}_{3}, 400 \mathrm{MHz}\right)$ 8: 7.33-7.19 (5H, m, ArH), $6.84(1 \mathrm{H}, \mathrm{s}, \mathrm{ArH}), 6.80$ $(1 \mathrm{H}, \mathrm{d}, J=8.5 \mathrm{~Hz}, \operatorname{ArH}), 6.76(1 \mathrm{H}, \mathrm{d}, J=8.2 \mathrm{~Hz}$, $\mathrm{ArH}), 5.87$ and $5.85(1 \mathrm{H}, 2 \mathrm{~s},=\mathrm{CH}), 4.92-4.84(2 \mathrm{H}$, m, 2"- and 2'-OCH), 3.80 and $3.79\left(6 \mathrm{H}, 2 \mathrm{~s}, 2 \times \mathrm{OCH}_{3}\right)$ $3.07(1 \mathrm{H}, \mathrm{dd}, J=15.1,8.7 \mathrm{~Hz}, 1 "-\mathrm{HCH}), 3.02-2.86$ $\left(3 \mathrm{H}, \mathrm{m}, 1^{\prime}-\mathrm{HCH}\right.$ and $\left.1^{\prime}-\mathrm{CH} 2\right) ;{ }^{13} \mathrm{C}$ NMR $\left(\mathrm{CDCl}_{3}\right) \delta$ : 35.9, 36.7, 55.8, 71.6, 72.4, 103.2, 108.9, 111.0,

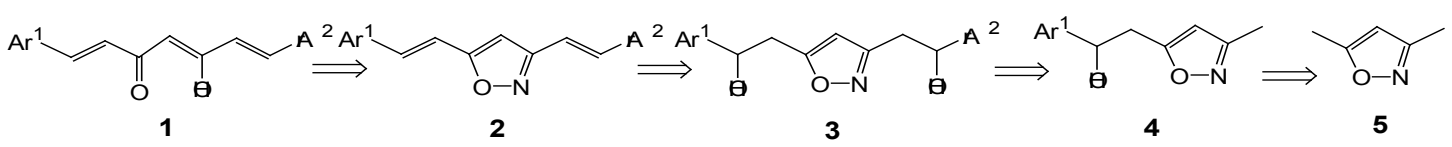

Scheme 1: 
$117.9,125.7,127.7,128.4,135.7,143.2,148.5$, $148.9,161.3,169.9$.

\section{3-[2-(4-Methoxyphenyl)-2-hydroxyethyl]-5-(2- (3,4-dimethoxyphenyl)-2-hydroxyethyl) isoxazole} (3f)

Yield: $0.74 \mathrm{~g}(46 \%)$; green crystals; $\mathrm{mp}$ $76-77^{\circ} \mathrm{C}$; IR (thin film) $v_{\max }: 3383,2935,1604,1513$, 1421, 1238, 1139, 1024, 811, $763 \mathrm{~cm}^{-1} ;{ }^{1} \mathrm{H}$ NMR $\left(\mathrm{CDCl}_{3}, 400 \mathrm{MHz}\right) \delta: 7.21(2 \mathrm{H}, \mathrm{d}, J=8.2 \mathrm{~Hz}, \mathrm{ArH})$, $6.85(1 \mathrm{H}, \mathrm{s}, \mathrm{ArH}), 6.84-6.79(3 \mathrm{H}, \mathrm{m}, \mathrm{ArH}), 6.77(1 \mathrm{H}$, $\mathrm{d}, J=8.2 \mathrm{~Hz}, \operatorname{ArH}), 5.89$ and $5.86(1 \mathrm{H}, 2 \mathrm{~s},=\mathrm{CH})$, 4.93-4.80 (2H, m, 2"- and 2'-OCH), 3.82 and 3.81 $\left(6 \mathrm{H}, 2 \mathrm{~s}, 2 \times \mathrm{OCH}_{3}\right), 3.75\left(3 \mathrm{H}, \mathrm{s}, \mathrm{OCH}_{3}\right), 3.08(1 \mathrm{H}, \mathrm{dd}$, $J=15.2$ and $8.7 \mathrm{~Hz}, 1 "-\mathrm{HCH}), 3.03-2.85(3 \mathrm{H}, \mathrm{m}$, $1 "-\mathrm{HCH}$ and 1 '- $\left.\mathrm{CH}_{2}\right) ;{ }^{13} \mathrm{C} \mathrm{NMR}\left(\mathrm{CDCl}_{3}\right) \delta: 35.9,36.7$, 55.2, 55.8, 55.9, 71.7, 72.1, 103.2, 108.9, 111.0, $113.8,117.9,127.0,135.4,135.8,148.5,149.0$, 159.1, 161.4, 169.9 .

General procedure for dehydration of 3,5-Bis(2aryl-2-hydroxyethyl) isoxazoles 3a-f

A mixture of 3,5-bis(2-aryl-2-hydroxyethyl) isoxazole $(1.0 \mathrm{mmol}), \mathrm{P}_{2} \mathrm{O}_{5}(0.4 \mathrm{mmol})$ in benzene $(5 \mathrm{ml})$ was heated under reflux temperature for 2 hours. The benzene layer was decanted and the residue was washed once with hot benzene. The combined benzene layer was washed with water, dried $\left(\mathrm{Na}_{2} \mathrm{SO}_{4}\right)$ and concentrated under reduced pressure. The residue was purified using column chromatography on silica gel with $\mathrm{CH}_{2} \mathrm{Cl}_{2}$ as eluent to give 5-(2-arylethenyl)-3-methylisoxazoles 2a-f (52-66\%) as crystalline solids.

\section{3,5-Bis(2-Phenylethenyl) isoxazole (2a)}

Yield: $0.18 \mathrm{~g}(66 \%)$; white crystals; $\mathrm{mp} 186-$ $187^{\circ} \mathrm{C}$; IR (thin film) $v_{\max }: 3033,1644,1579,1559$, 1429, 1292, 960, 751, $693 \mathrm{~cm}^{-1} ;{ }^{1} \mathrm{H}$ NMR $\left(\mathrm{CDCl}_{3}\right.$, $400 \mathrm{MHz}) \mathrm{d}: 7.56-7.48(4 \mathrm{H}, \mathrm{m}, \mathrm{ArH}), 7.42-7.29(7 \mathrm{H}$, $\mathrm{m}, \mathrm{ArH}$ and $\left.\mathrm{Ar}^{1} \mathrm{CH}=\mathrm{CH}-\right), 7.19(1 \mathrm{H}, \mathrm{d}, J=16.6 \mathrm{~Hz}$, $\left.\mathrm{Ar}^{2} \mathrm{CH}=\mathrm{CH}-\right), 7.13\left(1 \mathrm{H}, \mathrm{d}, J=16.5 \mathrm{~Hz}, \mathrm{Ar}^{2} \mathrm{CH}=\mathrm{CH}-\right)$, $6.96\left(1 \mathrm{H}, \mathrm{d}, \mathrm{J}=16.4 \mathrm{~Hz}, \mathrm{Ar}^{1} \mathrm{CH}=\mathrm{CH}-\right), 6.49(1 \mathrm{H}, \mathrm{s}$,
$=\mathrm{CH}) ;{ }^{13} \mathrm{C} \mathrm{NMR}\left(\mathrm{CDCl}_{3}\right) \delta: 98.5,113.0,116.1,127.0$, $127.1,128.9,129.2,134.9,135.5,135.8,162.0$, 168.3.

\section{3,5-Bis(2-(4-methoxyphenyl)ethenyl)isoxazole} (2b)

Yield: $0.20 \mathrm{~g}(61 \%) ;$ yellow crystals; mp 194$196{ }^{\circ} \mathrm{C}$ (lit. ${ }^{11} 180-181^{\circ} \mathrm{C}$ ); IR (thin film) $v_{\max }: 2934$, 1644, 1604, 1577, 1512, 1431, 1257, 1175, 1029 , 967, 823, $753 \mathrm{~cm}^{-1} ;{ }^{1} \mathrm{H} \mathrm{NMR}\left(\mathrm{CDCl}_{3}, 400 \mathrm{MHz}\right) \delta: 7.48$ $(4 \mathrm{H}, \mathrm{d}, J=8.7 \mathrm{~Hz}, \mathrm{ArH}), 7.31(1 \mathrm{H}, \mathrm{d}, J=16.4 \mathrm{~Hz}$, $\left.\operatorname{Ar}^{1} \mathrm{CH}=\mathrm{CH}-\right), 7.13\left(1 \mathrm{H}, \mathrm{d}, J=16.5 \mathrm{~Hz}, \mathrm{Ar}^{2} \mathrm{CH}=\mathrm{CH}-\right)$, $7.00\left(1 \mathrm{H}, \mathrm{d}, J=16.5 \mathrm{~Hz}, \mathrm{Ar}^{2} \mathrm{CH}=\mathrm{CH}-\right), 6.92(4 \mathrm{H}, \mathrm{d}, J$ $=8.5 \mathrm{~Hz}, \mathrm{ArH}), 6.83\left(1 \mathrm{H}, \mathrm{d}, J=16.4 \mathrm{~Hz}, \mathrm{Ar}^{1} \mathrm{CH}=\mathrm{CH}-\right)$, $6.43(1 \mathrm{H}, \mathrm{s},=\mathrm{CH}), 3.84\left(6 \mathrm{H}, \mathrm{s}, 2 \times \mathrm{OCH}_{3}\right) ;{ }^{13} \mathrm{C} \mathrm{NMR}$

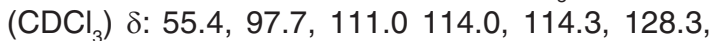
$128.6,128.7,134.4,135.2,160.2,160.5,162.2$, 168.5 .

\section{3,5-Bis [2-(3,4-dimethoxyphenyl)ethenyl] isoxazole (2c)}

Yield: $0.22 \mathrm{~g}(56 \%) ;$ white crystals; mp 169$171{ }^{\circ} \mathrm{C}$ (lit. ${ }^{11} 159-160{ }^{\circ} \mathrm{C}$ ); IR (thin film) $v_{\max }: 2937$, 1644, 1584, 1561, 1512, 1428, 1264, 1139, 1024, 963, 805, $735 \mathrm{~cm}^{-1} ;{ }^{1} \mathrm{H}$ NMR $\left(\mathrm{CDCl}_{3}, 400 \mathrm{MHz}\right) \delta: 7.22$ $\left(1 \mathrm{H}, \mathrm{d}, J=16.4 \mathrm{~Hz}, \mathrm{Ar}^{1} \mathrm{CH}=\mathrm{CH}-\right), 7.09-6.93(6 \mathrm{H}, \mathrm{m}$, $\mathrm{ArH}, \mathrm{Ar}^{2} \mathrm{CH}=\mathrm{CH}$ - and $\left.\mathrm{Ar}^{2} \mathrm{CH}=\mathrm{CH}-\right), 6.83-6.73(3 \mathrm{H}$, $\mathrm{m}, \mathrm{ArH}$ and $\left.\mathrm{Ar}^{1} \mathrm{CH}=\mathrm{CH}-\right), 6.36(1 \mathrm{H}, \mathrm{s},=\mathrm{CH}), 3.90$ and $3.89\left(6 \mathrm{H}, 2 \mathrm{~s}, 2 \times \mathrm{OCH}_{3}\right), 3.86$ and $3.85(6 \mathrm{H}, 2 \mathrm{~s}$, $\left.2 \mathrm{xOCH}_{3}\right) ;{ }^{13} \mathrm{C} \mathrm{NMR}\left(\mathrm{CDCl}_{3}\right) \delta: 55.8,97.8,108.8$, $109.1,110.0,111.1,113.9,120.9,121.0,128.5$, $128.8,134.5,135.4,149.1,149.8,150.1,162.1$ 168.4 .

5-(2 - (4 - Methoxyphenyl)ethenyl)-3-[2 phenylethenyl] isoxazole (2d)

Yield: $0.18 \mathrm{~g}(60 \%)$; yellow crystals; $\mathrm{mp}$ $155-156^{\circ} \mathrm{C}$; IR (thin film) $v_{\max }: 3035,2928,1643$, 1603, 1509, 1430, 1254, 1173, 1032, 963, 822, 754, $695 \mathrm{~cm}^{-1} ;{ }^{1} \mathrm{H} \mathrm{NMR}\left(\mathrm{CDCl}_{3}, 400 \mathrm{MHz}\right) \delta: 7.53(2 \mathrm{H}$, d, $J=7.2 \mathrm{~Hz}, \operatorname{ArH}), 7.47(2 \mathrm{H}, \mathrm{d}, J=8.7 \mathrm{~Hz}, \operatorname{ArH})$,

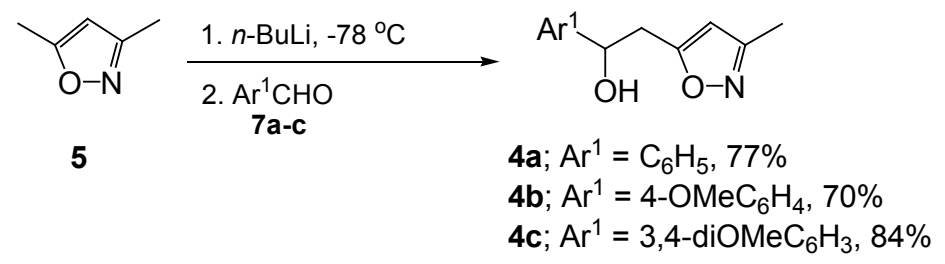

Scheme 2: 
7.42-7.28 (4H, m, ArH and $\left.\mathrm{Ar}^{1} \mathrm{CH}=\mathrm{CH}-\right), 7.18(1 \mathrm{H}$, $\left.\mathrm{d}, J=16.6 \mathrm{~Hz}, \mathrm{Ar}^{2} \mathrm{CH}=\mathrm{CH}-\right), 7.13(1 \mathrm{H}, \mathrm{d}, J=16.5$ $\left.\mathrm{Hz}, \mathrm{Ar}^{2} \mathrm{CH}=\mathrm{CH}-\right), 6.92(2 \mathrm{H}, \mathrm{d}, J=8.7 \mathrm{~Hz}, \mathrm{ArH}), 6.83$ $\left(1 \mathrm{H}, \mathrm{d}, J=16.4 \mathrm{~Hz}, \mathrm{Ar}^{1} \mathrm{CH}=\mathrm{CH}-\right), 6.44(1 \mathrm{H}, \mathrm{s},=\mathrm{CH})$, $3.84\left(3 \mathrm{H}, \mathrm{s}, \mathrm{OCH}_{3}\right) ;{ }^{13} \mathrm{C} \mathrm{NMR}\left(\mathrm{CDCl}_{3}\right) \delta: 55.4,97.7$, $110.9,114.3,116.2,127.0,128.3,128.6,128.8$, $135.5,135.6,135.9,160.5,162.0,168.7$.

\section{5-(2-(3,4-Dimethoxyphenyl)ethenyl)-3-[2-} phenylethenyl]isoxazole (2e)

Yield: $0.19 \mathrm{~g} \mathrm{(58 \% );} \mathrm{white} \mathrm{crystals;} \mathrm{mp}$ 153-154 ${ }^{\circ} \mathrm{C}$; IR (thin film) $v_{\text {max }}: 2936,1644,1583$, 1561, 1513, 1433, 1269, 1140, 1025, 964, 754, $697 \mathrm{~cm}^{-1} ;{ }^{1} \mathrm{H}$ NMR $\left(\mathrm{CDCl}_{3}, 400 \mathrm{MHz}\right) \delta: 7.47(2 \mathrm{H}$, d, $J=7.5 \mathrm{~Hz}, \mathrm{ArH}), 7.39-7.28(3 \mathrm{H}, \mathrm{m}, \mathrm{ArH}), 7.25$ $\left(1 \mathrm{H}, \mathrm{d}, J=16.4 \mathrm{~Hz}, \mathrm{Ar}^{1} \mathrm{CH}=\mathrm{CH}-\right), 7.13(1 \mathrm{H}, \mathrm{d}, J$ $\left.=16.6 \mathrm{~Hz}, \mathrm{Ar}^{2} \mathrm{CH}=\mathrm{CH}-\right), 7.08(1 \mathrm{H}, \mathrm{d}, J=16.6 \mathrm{~Hz}$, $\left.\mathrm{Ar}^{2} \mathrm{CH}=\mathrm{CH}-\right), 7.05-6.98(2 \mathrm{H}, \mathrm{m}, \mathrm{ArH}), 6.78(2 \mathrm{H}, \mathrm{m}$, $\mathrm{ArH}$ and $\left.\mathrm{Ar}^{1} \mathrm{CH}=\mathrm{CH}-\right), 6.39(1 \mathrm{H}, \mathrm{s},=\mathrm{CH}), 3.90(3 \mathrm{H}$, $\left.\mathrm{s}, \mathrm{OCH}_{3}\right), 3.85\left(3 \mathrm{H}, \mathrm{s}, \mathrm{OCH}_{3}\right) ;{ }^{13} \mathrm{C} \mathrm{NMR}\left(\mathrm{CDCl}_{3}\right) \delta$ : 55.8, 55.9, 97.9, 109.1, 111.0, 111.2, 116.1, 121.1, $126.9,128.5,128.8,134.7,135.6,135.8,149.2$, $150.1,161.9,168.5$.

5-(2-(3,4-Dimethoxyphenyl)ethenyl)-3-[2-(4methoxyphenyl)ethenyl] isoxazole (2f)

Yield: $0.19 \mathrm{~g}$ (52\%); white crystals; $\mathrm{mp}$ $169-171^{\circ} \mathrm{C}$; IR (thin film) $v_{\text {max }}$ : 2962, 1643, 1603, 1581, 1512, 1430, 1264, 1174, 1140, 1026, 965, $821,750 \mathrm{~cm}^{-1} ;{ }^{1} \mathrm{H}$ NMR $\left(\mathrm{CDCl}_{3}, 400 \mathrm{MHz}\right) \delta: 7.45$ $(2 \mathrm{H}, \mathrm{d}, J=8.6 \mathrm{~Hz}, \mathrm{ArH}), 7.27(1 \mathrm{H}, \mathrm{d}, J=16.4 \mathrm{~Hz}$, $\left.\operatorname{Ar}^{1} \mathrm{CH}=\mathrm{CH}-\right), 7.10\left(1 \mathrm{H}, \mathrm{d}, J=16.6 \mathrm{~Hz}, \mathrm{Ar}^{2} \mathrm{CH}=\mathrm{CH}-\right)$, $7.05(2 \mathrm{H}, \mathrm{d}, J=7.7 \mathrm{~Hz}, \mathrm{ArH}), 6.98(1 \mathrm{H}, \mathrm{d}, J=16.4$ $\left.\mathrm{Hz}, \mathrm{Ar}^{2} \mathrm{CH}=\mathrm{CH}-\right), 6.93-6.84(3 \mathrm{H}, \mathrm{m}, \mathrm{ArH}), 6.81(1 \mathrm{H}$, $\left.\mathrm{d}, J=16.4 \mathrm{~Hz}, \mathrm{Ar}^{1} \mathrm{CH}=\mathrm{CH}-\right), 6.40(1 \mathrm{H}, \mathrm{s},=\mathrm{CH})$, $3.93\left(3 \mathrm{H}, \mathrm{s}, \mathrm{OCH}_{3}\right), 3.90\left(3 \mathrm{H}, \mathrm{s}, \mathrm{OCH}_{3}\right), 3.82(3 \mathrm{H}, \mathrm{s}$, $\left.\mathrm{OCH}_{3}\right) ;{ }^{13} \mathrm{C} \mathrm{NMR}\left(\mathrm{CDCl}_{3}\right) \delta: 55.3,55.9,97.8,109.1$, $111.1,111.2,113.9,114.3,121.1,128.3,128.6$, 134.6, 135.2, 149.2, 150.2, 160.2, 162.2, 168.4 .

General procedure for reductive ring opening of 3,5-bis(2-arylethenyl) isoxazoles $2 a-f$

A solution of a 3,5-bis(2-arylethenyl) isoxazole $(0.4 \mathrm{mmol})$, water $(0.4 \mathrm{mmol})$ and $\left[\mathrm{Mo}(\mathrm{CO})_{6}\right](0.2 \mathrm{mmol})$ in acetonitrile $(8 \mathrm{ml})$ was heated under reflux temperature for 24 hours and cooled to room temperature. The reaction mixture was filtered through Celite and evaporated to dryness under reduced pressure. Purification of the residue using column chromatography on silica gel with $30 \%$ ethyl acetate/ hexane as an eluent gave1,7-diaryl-5-amino-1,4,6-heptatrien-3-ones 8a-f $(43-56 \%)$ as yellow crystalline solids or as yellow oils.

\section{5-Amino-1,7-diphenylhepta-1,4,6-trien-3-one} (8a)

Yield: $61.5 \mathrm{mg}$ (56\%); yellow crystals; mp $145-147^{\circ} \mathrm{C}$; IR (thin film) $v_{\max }: 3388,3196,1634$, 1570, 1519, 1149, 966, 754, $694 \mathrm{~cm}^{-1} ;{ }^{1} \mathrm{H}$ NMR $\left(\mathrm{CDCl}_{3}, 400 \mathrm{MHz}\right) \delta: 7.60-7.54(3 \mathrm{H}, \mathrm{m}, \mathrm{ArH}$ and $\left.\operatorname{Ar}^{1} \mathrm{CH}=\mathrm{CH}-\right), 7.49(2 \mathrm{H}, \mathrm{d}, J=7.1 \mathrm{~Hz}, \mathrm{ArH}) 7.41-7.30$ $(6 \mathrm{H}, \mathrm{m}, \mathrm{ArH}), 7.11\left(1 \mathrm{H}, \mathrm{d}, J=16.4 \mathrm{~Hz}, \mathrm{Ar}^{2} \mathrm{CH}=\mathrm{CH}-\right)$, $6.79\left(1 \mathrm{H}, \mathrm{d}, J=15.9 \mathrm{~Hz}, \mathrm{Ar}^{2} \mathrm{CH}=\mathrm{CH}-\right), 6.53(1 \mathrm{H}, \mathrm{d}$, $\left.J=16.4 \mathrm{~Hz}, \mathrm{Ar}^{1} \mathrm{CH}=\mathrm{CH}-\right), 5.53(1 \mathrm{H}, \mathrm{s},=\mathrm{CHCO}) ;{ }^{13} \mathrm{C}$ NMR $\left(\mathrm{CDCl}_{3}\right) \delta: 98.7,124.8,127.4,127.9,128.6$, $128.8,129.0,129.4,129.5,134.5,135.2,135.7$, $138.7,157.7,188.4$.

\section{5-Amino-1,7-bis(4-methoxyphenyl)-1,4,6- heptatrien-3-one $(8 b)$}

Yield: $66.8 \mathrm{mg}(50 \%) ;$ yellow crystals; $\mathrm{mp}$ $158-160^{\circ} \mathrm{C}$; IR (thin film) $v_{\max }: 3393,3006,1630$, 1601, 1565, 1515, 1256, 1174, $754 \mathrm{~cm}^{-1} ;{ }^{1} \mathrm{H}$ NMR $\left(\mathrm{CDCl}_{3}, 400 \mathrm{MHz}\right) \delta: 7.56-7.47(3 \mathrm{H}, \mathrm{m}, \mathrm{ArH}$ and $\left.\operatorname{Ar}^{1} \mathrm{CH}=\mathrm{CH}-\right), 7.43(2 \mathrm{H}, \mathrm{d}, J=8.5 \mathrm{~Hz}, \mathrm{ArH}), 7.05(1 \mathrm{H}$, $\left.\mathrm{d}, J=16.3 \mathrm{~Hz}, \mathrm{Ar}^{2} \mathrm{CH}=\mathrm{CH}-\right), 6.93-6.86(4 \mathrm{H}, \mathrm{m}, \mathrm{ArH})$, $6.66\left(1 \mathrm{H}, \mathrm{d}, J=15.8 \mathrm{~Hz}, \mathrm{Ar}^{2} \mathrm{CH}=\mathrm{CH}-\right), 6.39(1 \mathrm{H}, \mathrm{d}, J$ $\left.=16.3 \mathrm{~Hz}, \mathrm{Ar}^{1} \mathrm{CH}=\mathrm{CH}-\right), 5.47(1 \mathrm{H}, \mathrm{s},=\mathrm{CHCO}), 3.83$ $\left(6 \mathrm{H}, \mathrm{s}, 2 \times \mathrm{OCH}_{3}\right) ;{ }^{13} \mathrm{C} \mathrm{NMR}\left(\mathrm{CDCl}_{3}\right) \delta: 55.3,55.4,98.4$, $114.2,114.4,122.4,126.6,128.0,128.5,128.8$, $129.4,134.0,138.2,158.1,160.7,188.3$.

\section{5-Amino-1,7-bis(3,4-dimethoxyphenyl)-1,4,6- heptatrien-3-one (8c)}

Yield: $76.2 \mathrm{mg}$ (48\%); yellow oil; IR (thin film) $v_{\max }: 3417,2937,1629,1587,1513,1461,1263$, 1139, 1024, $758 \mathrm{~cm}^{-1} ;{ }^{1} \mathrm{H}$ NMR $\left(\mathrm{CDCl}_{3}, 400 \mathrm{MHz}\right)$ $\delta: 7.51\left(1 \mathrm{H}, \mathrm{d}, J=15.7 \mathrm{~Hz}, \mathrm{Ar}^{1} \mathrm{CH}=\mathrm{CH}-\right), 7.14-7.01$ $\left(5 \mathrm{H}, \mathrm{m}, \mathrm{ArH}\right.$ and $\left.\mathrm{Ar}^{2} \mathrm{CH}=\mathrm{CH}-\right), 6.85(2 \mathrm{H}, \mathrm{d}, J=8.3$ $\mathrm{Hz}, \mathrm{ArH}), 6.67\left(1 \mathrm{H}, \mathrm{d}, J=15.8 \mathrm{~Hz}, \mathrm{Ar}^{2} \mathrm{CH}=\mathrm{CH}-\right)$, $6.39\left(1 \mathrm{H}, \mathrm{d}, J=16.3 \mathrm{~Hz}, \mathrm{Ar}^{1} \mathrm{CH}=\mathrm{CH}-\right), 5.52(1 \mathrm{H}, \mathrm{s}$, $=\mathrm{CHCO}), 3.92$ and $3.90\left(12 \mathrm{H}, 2 \mathrm{~s}, 4 \times \mathrm{OCH}_{3}\right) ;{ }^{13} \mathrm{C} \mathrm{NMR}$ $\left(\mathrm{CDCl}_{3}\right) \delta:$ 55.9, 56.0, 98.3, 109.3, 109.7, 111.1, $111.2,121.5,122.2,122.6,126.7,128.3,128.8$, 134.4, 138.5, 149.1, 149.3, 150.4, 158.1, 188.2.

\section{5-Amino-1-(4-methoxyphenyl)-7-phenyl-1,4,6- heptatrien-3-one (8d)}

Yield: $66.0 \mathrm{mg}$ (54\%); yellow crystals; mp 161-162 ${ }^{\circ} \mathrm{C}$; IR (thin film) $v_{\max }: 3364,3008,1631$, 
1602, 1564, 1515, 1256, 1145, $752 \mathrm{~cm}^{-1} ;{ }^{1} \mathrm{H}$ NMR $\left(\mathrm{CDCl}_{3}, 400 \mathrm{MHz}\right) \delta: 7.56-7.45(5 \mathrm{H}, \mathrm{m}, \mathrm{ArH}$ and $\left.\mathrm{Ar}^{1} \mathrm{CH}=\mathrm{CH}-\right), 7.42-7.29(3 \mathrm{H}, \mathrm{m}, \mathrm{ArH}), 7.09(1 \mathrm{H}, \mathrm{d}, J$ $\left.=16.4 \mathrm{~Hz}, \mathrm{Ar}^{2} \mathrm{CH}=\mathrm{CH}-\right), 6.88(2 \mathrm{H}, \mathrm{d}, J=8.4 \mathrm{~Hz}, \mathrm{ArH})$, $6.67\left(1 \mathrm{H}, \mathrm{d}, J=15.8 \mathrm{~Hz}, \mathrm{Ar}^{2} \mathrm{CH}=\mathrm{CH}-\right), 6.51(1 \mathrm{H}, \mathrm{d}, J$ $\left.=16.4 \mathrm{~Hz}, \mathrm{Ar}^{1} \mathrm{CH}=\mathrm{CH}-\right), 5.49(1 \mathrm{H}, \mathrm{s},=\mathrm{CHCO}), 3.81$ $\left(3 \mathrm{H}, \mathrm{s}, \mathrm{OCH}_{3}\right) ;{ }^{13} \mathrm{C} \mathrm{NMR}\left(\mathrm{CDCl}_{3}\right)$ : $: 55.3,98.8,114.2$, $124.9,126.5,127.3,128.4,128.9,129.3,129.5$, $134.3,135.3,138.5,157.5,160.8,188.6$.

\section{5-Amino-1-(3,4-dimethoxyphenyl)-7-phenyl-1,4,6-} heptatrien-3-one (8e)

Yield: $62.6 \mathrm{mg}$ (47\%); yellow oil; IR (thin film) $v_{\text {max }}: 3395,2959,1631,1575,1516,1264,1139,756$ $\mathrm{cm}^{-1} ;{ }^{1} \mathrm{H}$ NMR $\left(\mathrm{CDCl}_{3}, 400 \mathrm{MHz}\right)$ 8: 7.55-7.47 (3H, m, $\mathrm{ArH}$ and $\left.\mathrm{Ar}^{1} \mathrm{CH}=\mathrm{CH}-\right)$, 7.41-7.31 (3H, $\left.\mathrm{m}, \mathrm{Ar} H\right)$, 7.14$7.08\left(3 \mathrm{H}, \mathrm{m}, \mathrm{ArH}\right.$ and $\left.\mathrm{Ar}^{2} \mathrm{CH}=\mathrm{CH}-\right), 6.85(1 \mathrm{H}, \mathrm{d}, J=$ $8.3 \mathrm{~Hz}, \mathrm{ArH}), 6.68\left(1 \mathrm{H}, \mathrm{d}, J=15.8 \mathrm{~Hz}, \mathrm{Ar}^{2} \mathrm{CH}=\mathrm{CH}-\right)$, $6.52\left(2 \mathrm{H}, \mathrm{d}, J=16.4 \mathrm{~Hz}, \mathrm{Ar}^{1} \mathrm{CH}=\mathrm{CH}-\right), 5.53(1 \mathrm{H}, \mathrm{s}$, $=\mathrm{CHCO}), 3.92\left(3 \mathrm{H}, \mathrm{s}, \mathrm{OCH}_{3}\right), 3.90\left(3 \mathrm{H}, \mathrm{s}, \mathrm{OCH}_{3}\right) ;{ }^{13} \mathrm{C}$ $\operatorname{NMR}\left(\mathrm{CDCl}_{3}\right)$ 8: 55.9, 56.0, 98.6, 109.8, 111.2, 122.2, $124.9,126.7,127.3,128.7,128.9,129.4,134.3$, 135.3, 138.7, 149.2, 150.5, 157.5, 188.5 .
5-Amino-1-(3,4-dimethoxyphenyl)-7-(4methoxyphenyl)-1,4,6-heptatrien-3-one (8f)

Yield: $62.7 \mathrm{mg}$ (43\%); yellow oil; IR (thin film) $v_{\text {max }}: 3394,2964,1597,1514,1262,1141,1027,755$ $\mathrm{cm}^{-1} ;{ }^{1} \mathrm{H}$ NMR $\left(\mathrm{CDCl}_{3}, 400 \mathrm{MHz}\right) \delta: 7.51(1 \mathrm{H}, \mathrm{d}, J=$ $\left.15.7 \mathrm{~Hz}, \mathrm{Ar}^{1} \mathrm{CH}=\mathrm{CH}-\right), 7.43(2 \mathrm{H}, \mathrm{d}, J=8.6 \mathrm{~Hz}, \mathrm{ArH})$, 7.15-7.02 (3H, m, $\mathrm{ArH}$ and $\left.\mathrm{Ar}^{2} \mathrm{CH}=\mathrm{CH}-\right), 6.92-6.82$ $(3 \mathrm{H}, \mathrm{m}, \mathrm{ArH}), 6.67\left(1 \mathrm{H}, \mathrm{d}, J=15.7 \mathrm{~Hz}, \mathrm{Ar}^{2} \mathrm{CH}=\mathrm{CH}-\right)$, $6.39\left(1 \mathrm{H}, \mathrm{d}, J=16.3 \mathrm{~Hz}, \mathrm{Ar}^{1} \mathrm{CH}=\mathrm{CH}-\right), 5.50(1 \mathrm{H}, \mathrm{s}$, $=\mathrm{CHCO}), 3.92\left(3 \mathrm{H}, \mathrm{s}, \mathrm{OCH}_{3}\right), 3.90\left(3 \mathrm{H}, \mathrm{s}, \mathrm{OCH}_{3}\right)$, $3.83\left(3 \mathrm{H}, \mathrm{s}, \mathrm{OCH}_{3}\right) ;{ }^{13} \mathrm{C} \mathrm{NMR}\left(\mathrm{CDCl}_{3}\right) \delta: 55.3,55.9$, 98.1, 109.7, 111.1, 114.4, 122.2, 122.3, 126.9, 128.0, $128.8,134.2,138.4,149.1,150.4,158.3,160.7$, 188.1.

General procedure for hydrolysis of 5-amino-1,7diaryl-1,4,6-heptatrien-3-ones 8a-f

To a stirred solution of 5-amino-1-aryl1,4-hexadien-3-one $(0.1 \mathrm{mmol})$ in ethanol $(1.5 \mathrm{ml})$ was added concentrated $\mathrm{HCl}$ dropwise to adjust the $\mathrm{pH}$ between 4 and 5 . The solution was then stirred at $50^{\circ} \mathrm{C}$. After 24 hours, the reaction mixture was neutralized with a saturated $\mathrm{K}_{2} \mathrm{CO}_{3}$ solution.<smiles>Cc1cc(CC(O)Br)on1</smiles>

4a-c

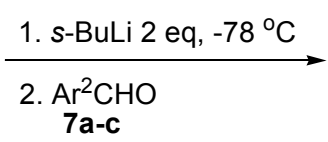<smiles>OC([Al])[C]c1cc([CH]C(O)[Al])no1</smiles>

3a; $\mathrm{Ar}^{1}=\mathrm{C}_{6} \mathrm{H}_{5}, \mathrm{Ar}^{2}=\mathrm{C}_{6} \mathrm{H}_{5}, 48 \%$

3b; $\mathrm{Ar}^{1}=4-\mathrm{OMeC}_{6} \mathrm{H}_{4}, \mathrm{Ar}^{2}=4-\mathrm{OMeC}_{6} \mathrm{H}_{4}, 49 \%$

3c; $\mathrm{Ar}^{1}=3,4-\mathrm{diOMeC}_{6} \mathrm{H}_{3}, \mathrm{Ar}^{2}=3,4-\mathrm{diOMeC}_{6} \mathrm{H}_{3}, 65 \%$

3d; $\mathrm{Ar}^{1}=4-\mathrm{OMeC}_{6} \mathrm{H}_{4}, \mathrm{Ar}^{2}=\mathrm{C}_{6} \mathrm{H}_{5}, 57 \%$

3e; $\mathrm{Ar}^{1}=3,4-\mathrm{diOMeC}_{6} \mathrm{H}_{3}, \mathrm{Ar}^{2}=\mathrm{C}_{6} \mathrm{H}_{5}, 51 \%$

3f; $\mathrm{Ar}^{1}=3,4-\mathrm{diOMeC}_{6} \mathrm{H}_{3}, \mathrm{Ar}^{2}=4-\mathrm{OMeC}_{6} \mathrm{H}_{4}, 46 \%$

Scheme 3:<smiles>[Y10]C(O)Cc1cc(CC(O)[Al])on1</smiles><smiles>[Te]=C=[Te]</smiles>

2a; $A r^{1}=\mathrm{C}_{6} \mathrm{H}_{5}, \mathrm{Ar}^{2}=\mathrm{C}_{6} \mathrm{H}_{5}, 66 \%$

2b; $\mathrm{Ar}^{1}=4-\mathrm{OMeC}_{6} \mathrm{H}_{4}, \mathrm{Ar}^{2}=4-\mathrm{OMeC}_{6} \mathrm{H}_{4}, 61 \%$

2c; $\mathrm{Ar}^{1}=3,4-\mathrm{diOMeC}_{6} \mathrm{H}_{3}, \mathrm{Ar}^{2}=3,4-\mathrm{diOMeC}_{6} \mathrm{H}_{3}, 56 \%$

2d; $\mathrm{Ar}^{1}=4-\mathrm{OMeC}_{6} \mathrm{H}_{4}, \mathrm{Ar}^{2}=\mathrm{C}_{6} \mathrm{H}_{5}, 60 \%$

2e; $\mathrm{Ar}^{1}=3,4-\mathrm{diOMeC}_{6} \mathrm{H}_{3}, \mathrm{Ar}^{2}=\mathrm{C}_{6} \mathrm{H}_{5}, 58 \%$

2f; $\mathrm{Ar}^{1}=3,4-\mathrm{diOMeC}_{6} \mathrm{H}_{3}, \mathrm{Ar}^{2}=4-\mathrm{OMeC}_{6} \mathrm{H}_{4}, 52 \%$

Scheme 4: 
The aqueous layer was extracted with diethyl ether $(3 \times 25 \mathrm{~mL})$. The combined organic layer was dried $\left(\mathrm{Na}_{2} \mathrm{SO}_{4}\right)$ and concentrated under reduce pressure. Purification of the residue using column chromatography on silica gel with a gradient of $30-50 \%$ ethyl acetate/hexane as eluent gave 1-aryl5-hydroxy-1,4-hexadien-3-ones 1a-f (48-63\%) as crystalline solids.

\section{5-Hydroxy-1,7-diphenylhepta-1,4,6-trien-3-one} (1a)

Yield: $14.4 \mathrm{mg}$ (52\%); yellow crystals; $\mathrm{mp}$ $146-148^{\circ} \mathrm{C}$; IR (thin film) $v_{\max }: 3057,1627,1581$, 1445, 1275, 1139, 972, $753 \mathrm{~cm}^{-1}$; ${ }^{1} \mathrm{H} \mathrm{NMR}\left(\mathrm{CDCl}_{3}\right.$, $400 \mathrm{MHz}) \delta: 7.67\left(2 \mathrm{H}, \mathrm{d}, J=15.9 \mathrm{~Hz}, \mathrm{Ar}^{1} \mathrm{CH}=\mathrm{CH}-\right.$ and $\left.\mathrm{Ar}^{2} \mathrm{CH}=\mathrm{CH}-\right), 7.56(4 \mathrm{H}$, dd, $J=7.4$ and 1.8 $\mathrm{Hz}, \mathrm{ArH}), 7.44-7.34(6 \mathrm{H}, \mathrm{m}, \mathrm{ArH}), 6.64(2 \mathrm{H}, \mathrm{d}, J=$ $15.9 \mathrm{~Hz}, \mathrm{Ar}^{2} \mathrm{CH}=\mathrm{CH}$ - and $\left.\mathrm{Ar}^{1} \mathrm{CH}=\mathrm{CH}-\right), 5.86(1 \mathrm{H}, \mathrm{s}$, $=\mathrm{CHCO}) ;{ }^{13} \mathrm{C} \mathrm{NMR}\left(\mathrm{CDCl}_{3}\right) \delta: 101.8,124.1,128.1$, 128.9, 130.1, 135.0, 140.6, 183.3.

5-Hydroxy-1,7-Bis(4-methoxyphenyl)-1,4,6heptatrien-3-one (1b)

Yield: $21.2 \mathrm{mg}$ (63\%); orange crystals;mp $175-177^{\circ} \mathrm{C}$; IR (thin film) $v_{\max }: 3037,2933,2838$,
1628, 1600, 1574, 1512, 1461, 1253, 1173, 1138, 1031, 974, $828 \mathrm{~cm}^{-1} ;{ }^{1} \mathrm{H}$ NMR $\left(\mathrm{CDCl}_{3}, 400 \mathrm{MHz}\right)$ d: $7.62\left(2 \mathrm{H}, \mathrm{d}, J=15.8 \mathrm{~Hz}, \mathrm{Ar}^{1} \mathrm{CH}=\mathrm{CH}-\right.$ and $\left.\mathrm{Ar}^{2} \mathrm{CH}=\mathrm{CH}-\right), 7.50(4 \mathrm{H}, \mathrm{d}, J=8.7 \mathrm{~Hz}, \mathrm{ArH}), 6.91$ $(4 \mathrm{H}, \mathrm{d}, J=8.7 \mathrm{~Hz}, \mathrm{ArH}), 6.49(2 \mathrm{H}, \mathrm{d}, J=15.8 \mathrm{~Hz}$, $\mathrm{Ar}^{2} \mathrm{CH}=\mathrm{CH}$ - and $\left.\mathrm{Ar}^{1} \mathrm{CH}=\mathrm{CH}-\right), 5.78(1 \mathrm{H}, \mathrm{s},=\mathrm{CHCO})$, $3.84\left(6 \mathrm{H}, \mathrm{s}, 2 \times \mathrm{OCH}_{3}\right) ;{ }^{13} \mathrm{C} \mathrm{NMR}\left(\mathrm{CDCl}_{3}\right) \delta: 55.4$, $101.3,114.4,121.8,127.8,129.8,140.1,161.3$, 183.3.

\section{5-Hydroxy-1,7-Bis(3,4-dimethoxyphenyl)-1,4,6- heptatrien-3-one (1c)}

Yield: $19.5 \mathrm{mg}$ (49\%); orange crystals; $\mathrm{mp} 115-117^{\circ} \mathrm{C}$; IR (thin film) $v_{\max }: 2935,2837,1624$, 1583, 1512, 1462, 1262, 1136, 1023, 969, $808 \mathrm{~cm}^{-1}$; ${ }^{1} \mathrm{H} \mathrm{NMR}\left(\mathrm{CDCl}_{3}, 400 \mathrm{MHz}\right) \delta: 7.61(2 \mathrm{H}, \mathrm{d}, J=15.8$ $\mathrm{Hz}, \mathrm{Ar}^{1} \mathrm{CH}=\mathrm{CH}-$ and $\left.\mathrm{Ar}^{2} \mathrm{CH}=\mathrm{CH}-\right), 7.14(2 \mathrm{H}, \mathrm{d}, J$ $=8.3 \mathrm{~Hz}, \mathrm{ArH}), 7.08(2 \mathrm{H}, \mathrm{s}, \mathrm{ArH}), 6.88(2 \mathrm{H}, \mathrm{d}, J=$ 8.3 Hz, ArH), $6.50\left(2 \mathrm{H}, \mathrm{d}, J=15.8 \mathrm{~Hz}, \mathrm{Ar}^{2} \mathrm{CH}=\mathrm{CH}\right.$ and $\left.\mathrm{Ar}^{1} \mathrm{CH}=\mathrm{CH}-\right), 5.82(1 \mathrm{H}, \mathrm{s},=\mathrm{CH}), 3.93(6 \mathrm{H}, \mathrm{s}$, $\left.2 \mathrm{XOCH}_{3}\right), 3.92\left(6 \mathrm{H}, \mathrm{s}, 2 \times \mathrm{OCH}_{3}\right) ;{ }^{13} \mathrm{C} \mathrm{NMR}\left(\mathrm{CDCl}_{3}\right) \delta$ : $55.9,56.0,101.3,109.8,111.2,122.1,122.6,128.1$, $140.4,149.3,151.1,183.3$.<smiles>[Al]C=Cc1cc(C=C[Al])on1</smiles>

2a-f

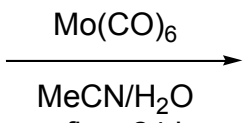
reflux, $24 \mathrm{~h}$

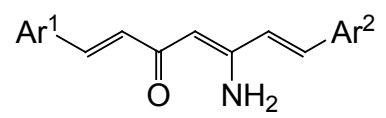

8a; $\mathrm{Ar}^{1}=\mathrm{C}_{6} \mathrm{H}_{5}, \mathrm{Ar}^{2}=\mathrm{C}_{6} \mathrm{H}_{5}, 56 \%$

8b; $\mathrm{Ar}^{1}=4-\mathrm{OMeC}_{6} \mathrm{H}_{4}, \mathrm{Ar}^{2}=4-\mathrm{OMeC}_{6} \mathrm{H}_{4}, 50 \%$

8c; $\mathrm{Ar}^{1}=3,4-$ diOMeC $_{6} \mathrm{H}_{3}, \mathrm{Ar}^{2}=3,4-\mathrm{diOMeC}_{6} \mathrm{H}_{3}, 48 \%$

8d; $\mathrm{Ar}^{1}=4-\mathrm{OMeC}_{6} \mathrm{H}_{4}, \mathrm{Ar}^{2}=\mathrm{C}_{6} \mathrm{H}_{5}, 54 \%$

8e; $\mathrm{Ar}^{1}=3,4-$ diOMeC $_{6} \mathrm{H}_{3}, \mathrm{Ar}^{2}=\mathrm{C}_{6} \mathrm{H}_{5}, 47 \%$

8f; $\mathrm{Ar}^{1}=3,4-\mathrm{diOMeC}_{6} \mathrm{H}_{3}, \mathrm{Ar}^{2}=4-\mathrm{OMeC}_{6} \mathrm{H}_{4}, 43 \%$

Scheme 5:<smiles>[R16][R5]([R5])([R5])[H]</smiles><smiles></smiles>

1a; $\mathrm{Ar}^{1}=\mathrm{C}_{6} \mathrm{H}_{5}, \mathrm{Ar}^{2}=\mathrm{C}_{6} \mathrm{H}_{5}, 52 \%$

1b; $\mathrm{Ar}^{1}=4-\mathrm{OMeC}_{6} \mathrm{H}_{4}, \mathrm{Ar}^{2}=4-\mathrm{OMeC}_{6} \mathrm{H}_{4}, 63 \%$

1c; $\mathrm{Ar}^{1}=3,4-\mathrm{diOMeC}_{6} \mathrm{H}_{3}, \mathrm{Ar}^{2}=3,4-\mathrm{diOMeC}_{6} \mathrm{H}_{3}, 49 \%$

1d; $\mathrm{Ar}^{1}=4-\mathrm{OMeC}_{6} \mathrm{H}_{4}, \mathrm{Ar}^{2}=\mathrm{C}_{6} \mathrm{H}_{5}, 48 \%$

1e; $\mathrm{Ar}^{1}=3,4-\mathrm{diOMeC}_{6} \mathrm{H}_{3}, \mathrm{Ar}^{2}=\mathrm{C}_{6} \mathrm{H}_{5}, 51 \%$

1f; $\mathrm{Ar}^{1}=3,4-\mathrm{diOMeC}_{6} \mathrm{H}_{3}, \mathrm{Ar}^{2}=4-\mathrm{OMeC}_{6} \mathrm{H}_{4}, 52 \%$

Scheme 6: 


\section{5-Hydroxy-1-(4-methoxyphenyl)-7-phenyl-1,4,6- heptatrien-3-one (1d)}

Yield: $14.7 \mathrm{mg} \mathrm{(48 \% );} \mathrm{yellow} \mathrm{crystals;} \mathrm{mp}$ $140-142^{\circ} \mathrm{C}$; IR (thin film) $v_{\max }$ : $3050,2933,2836$, $1623,1601,1576,1512,1447,1255,1175,1140$, 1028, 977, $826 \mathrm{~cm}^{-1} ;{ }^{1} \mathrm{H}$ NMR $\left(\mathrm{CDCl}_{3}, 400 \mathrm{MHz}\right)$ $\delta: 7.64\left(1 \mathrm{H}, \mathrm{d}, J=15.9 \mathrm{~Hz}, \mathrm{Ar}^{2} \mathrm{CH}=\mathrm{CH}-\right), 7.63(1 \mathrm{H}$, $\left.\mathrm{d}, J=15.8 \mathrm{~Hz}, \mathrm{Ar}^{1} \mathrm{CH}=\mathrm{CH}-\right), 7.55(2 \mathrm{H}, \mathrm{d}, J=7.5 \mathrm{~Hz}$, $\operatorname{ArH}), 7.51(2 \mathrm{H}, \mathrm{d}, J=8.7 \mathrm{~Hz}, \mathrm{ArH}), 7.43-7.35(3 \mathrm{H}$, $\mathrm{m}, \operatorname{Ar} H), 6.91(2 \mathrm{H}, \mathrm{d}, J=8.2 \mathrm{~Hz}, \operatorname{ArH}), 6.61(1 \mathrm{H}$, d, $\left.J=15.9 \mathrm{~Hz}, \mathrm{Ar}^{2} \mathrm{CH}=\mathrm{CH}-\right), 6.51(1 \mathrm{H}, \mathrm{d}, J=15.8$ $\left.\mathrm{Hz}, \mathrm{Ar}^{1} \mathrm{CH}=\mathrm{CH}-\right), 5.81(1 \mathrm{H}, \mathrm{s},=\mathrm{CHCO}), 3.83(3 \mathrm{H}$, $\left.\mathrm{s}, \mathrm{OCH}_{3}\right) ;{ }^{13} \mathrm{C} \mathrm{NMR}\left(\mathrm{CDCl}_{3}\right) \delta: 55.4,101.6,114.4$, $121.8,124.1,127.7,128.1,128.9,129.8,135.1$, $140.1,140.6,161.4,182.2,184.4$.

\section{5-Hydroxy-1-(3,4-dimethoxyphenyl)-7-phenyl- 1,4,6-heptatrien-3-one (1e)}

Yield: $17.1 \mathrm{mg}(51 \%)$; orange crystals; $\mathrm{mp}$ $138-139^{\circ} \mathrm{C}$; IR (thin film) $v_{\max }$ : $2934,2837,1624$, 1581, 1511, 1446, 1420, 1261, 1136, 1023, $968 \mathrm{~cm}^{-1}$; ${ }^{1} \mathrm{H} \mathrm{NMR}\left(\mathrm{CDCl}_{3}, 400 \mathrm{MHz}\right) \delta: 7.65(1 \mathrm{H}, \mathrm{d}, J=15.9 \mathrm{~Hz}$, $\left.\mathrm{Ar}^{2} \mathrm{CH}=\mathrm{CH}-\right), 7.62\left(1 \mathrm{H}, \mathrm{d}, J=15.7 \mathrm{~Hz}, \mathrm{Ar}^{1} \mathrm{CH}=\mathrm{CH}-\right)$, $7.55(2 \mathrm{H}, \mathrm{d}, J=7.6, \mathrm{ArH}), 7.43-7.34(3 \mathrm{H}, \mathrm{m}, \mathrm{ArH})$, $7.15(1 \mathrm{H}, \mathrm{d}, J=8.3 \mathrm{~Hz}, \operatorname{ArH}), 7.08(1 \mathrm{H}, \mathrm{s}, \operatorname{ArH}), 6.88$ $(1 \mathrm{H}, \mathrm{d}, J=8.3 \mathrm{~Hz}, \mathrm{Ar} H), 6.62(1 \mathrm{H}, \mathrm{d}, J=15.8 \mathrm{~Hz}$, $\left.\mathrm{Ar}^{2} \mathrm{CH}=\mathrm{CH}-\right), 6.52\left(1 \mathrm{H}, \mathrm{d}, J=15.8 \mathrm{~Hz}, \mathrm{Ar}^{1} \mathrm{CH}=\mathrm{CH}-\right)$, $5.84(1 \mathrm{H}, \mathrm{s},=\mathrm{CHCO}), 3.94\left(3 \mathrm{H}, \mathrm{s}, \mathrm{OCH}_{3}\right), 3.92(3$ $\left.\mathrm{H}, \mathrm{s}, \mathrm{OCH}_{3}\right) ;{ }^{13} \mathrm{C} \mathrm{NMR}\left(\mathrm{CDCl}_{3}\right) \delta: 55.9,56.0,101.5$, $109.9,111.2,122.1,122.8,124.1,128.0,128.1$, $128.9,130.0,135.1,140.2,140.8,149.3,151.2$, $182.4,184.2$.

\section{5-Hydroxy-1-(3,4-dimethoxyphenyl)-7-(4- methoxyphenyl)-1,4,6-heptatrien-3-one (1f)}

Yield: $19.0 \mathrm{mg}(52 \%)$; orange crystals; $\mathrm{mp}$ $155-157^{\circ} \mathrm{C}$; IR (thin film) $v_{\max }: 3002,2935,1625$, 1595, 1512, 1461, 1256, 1172, 1136, 1025, 970, $829 \mathrm{~cm}^{-1} ;{ }^{1} \mathrm{H}$ NMR $\left(\mathrm{CDCl}_{3}, 400 \mathrm{MHz}\right) \delta: 7.62(1 \mathrm{H}$, d, $\left.J=15.8 \mathrm{~Hz}, \mathrm{Ar}^{2} \mathrm{CH}=\mathrm{CH}-\right), 7.60(1 \mathrm{H}, \mathrm{d}, J=15.8$ $\left.\mathrm{Hz}, \mathrm{Ar}^{1} \mathrm{CH}=\mathrm{CH}-\right), 7.50(2 \mathrm{H}, \mathrm{d}, J=8.7 \mathrm{~Hz}, \mathrm{ArH}), 7.13$ $(1 \mathrm{H}, \mathrm{d}, J=8.3 \mathrm{~Hz}, \operatorname{ArH}), 7.08(1 \mathrm{H}, \mathrm{d}, J=1.5 \mathrm{~Hz}$, $\mathrm{ArH}), 6.91(2 \mathrm{H}, \mathrm{d}, J=8.7 \mathrm{~Hz}, \mathrm{ArH}), 6.87(1 \mathrm{H}, \mathrm{d}, J=$ $8.3 \mathrm{~Hz}, \mathrm{ArH}), 6.49\left(2 \mathrm{H}, \mathrm{d}, J=15.8 \mathrm{~Hz}, \mathrm{Ar}^{1} \mathrm{CH}=\mathrm{CH}-\right.$ and $\left.\mathrm{Ar}^{2} \mathrm{CH}=\mathrm{CH}-\right), 5.80(1 \mathrm{H}, \mathrm{s},=\mathrm{CHCO}), 3.93(3 \mathrm{H}, \mathrm{s}$, $\left.\mathrm{OCH}_{3}\right), 3.91\left(3 \mathrm{H}, \mathrm{s}, \mathrm{OCH}_{3}\right), 3.84\left(3 \mathrm{H}, \mathrm{s}, \mathrm{OCH}_{3}\right) ;{ }^{13} \mathrm{C}$ $\operatorname{NMR}\left(\mathrm{CDCl}_{3}\right) \delta: 55.4,55.9,56.0,101.3,109.8,111.2$, $114.4,121.8,122.1,122.6,127.8,128.1,129.8$, $140.2,140.3,149.3,151.1,161.3,183.1,183.4$.

\section{RESULTS AND DISCUSSION}

3,5-Dimethylisoxazole (5) used in our study was readily prepared in $82 \%$ yield from condensation between 2,4-pentanedione (6) with hydroxylamine hydrochloride in aqueous ethanol at reflux temperature for 3 hours. ${ }^{10}$

Lateral metalation of 3,5-dimethylisoxazole (5) occurred regiospecifically at the $\mathrm{C}_{5}$-methyl group upon treatment with either $n-\mathrm{BuLi}^{12}$ or $\mathrm{NaNH}_{2} \cdot{ }^{13}$ Metalation at the 3-methyl group could be accomplished when metalating agent was either $s$-BuLi or $t$-BuLi. ${ }^{14}$

As expected, lateral metalation of 3,5dimethylisoxazole (5) with $n$-BuLi in THF at $-78^{\circ} \mathrm{C}$ for 1 hour followed by quenching with aromatic aldehydes 7a-c gave the corresponding 5-(2-aryl-2hydroxyethyl)-3-methylisoxazoles $4 a-c$ in good yields (Scheme 2).

Further treatment of 5-(2-aryl-2hydroxyethyl)-3-methylisoxazoles $4 a-c$ with 2 equivalents of $s$-BuLi at $-78^{\circ} \mathrm{C}$ followed by quenching with aromatic aldehydes $7 \mathrm{a}-\mathrm{c}$ led to the formation of four diastereomers of the corresponding 3,5-bis(2aryl-2-hydroxyethyl)isoxazoles $\mathbf{3 a - f}$ in moderate yields (Scheme 3 ).

Since $\mathrm{P}_{2} \mathrm{O}_{5}$ is a very efficient dehydrating agent, it was used as such in dehydration of 3,5bis(2-aryl-2-hydroxyethyl)isoxazoles 3a-f. In benzene at reflux temperature ${ }^{15}$ (Scheme 4), the reaction between carbinols $3 a-f$ and $\mathrm{P}_{2} \mathrm{O}_{5}$ furnished the corresponding 3,5-bis(2-arylethenyl) isoxazoles 2a-f in moderate yields.

The weak $\mathrm{N}-\mathrm{O}$ bond of isoxazole ring is easily cleaved into $\beta$-aminoenone by either catalytic hydrogenolysis with platinum or palladium and Raney nickel under normal pressure and temperature ${ }^{16,17}$ or by treatment with transition metal carbonyls such as molybdenum hexacarbonyl $\left[\mathrm{Mo}(\mathrm{CO})_{6}\right] .^{18}$ To avoid catalytic hydrogenation at the two styryl groups, 3,5-bisstyrylisoxazoles 2a-f were treated with molybdenum hexacarbonyl in moist acetonitrile at reflux temperature. This treatment successfully provided the corresponding 1,7-diaryl-5-amino-1,4,6- 
heptatrien-3-ones 8a-f in moderate yields ( Scheme 5).

$\beta$-Aminoenones are easily transformed into $\beta$-hydroxyenones by simple acidic hydrolysis. ${ }^{19,20}$ In our final step, hydrolysis of $\beta$-aminoenones 8a-f was carried out by treatment with hydrochloric acid in ethanol at $\mathrm{pH} 4-5$. The corresponding curcumin derivatives, in their enol forms 1a-f were obtained in satisfied yields (Scheme 6).

\section{CONCLUSIONS}

The present six steps procedure provides a novel alternative route to symmetrical and unsymmetrical curcumin derivatives. Good to moderate yields of expected products were obtained from each step.

\section{ACKNOWLEDGEMENT}

We are pleased to acknowledge financial support from the Natural Products Research Unit, Department of Chemistry, Faculty of Science, Khon Kaen University, and the Center for Innovation in Chemistry (PERCH-CIC) and the Commission of Higher Education (CHE-RG), Ministry of Education.

\section{REFERENCES}

1. Anand, P.; Thomas, S.G.; Kunnumakkara, A.B.;Sundaram, C.;Harikumar, K.B.; Sung, B.; et al. Biochem. Pharmacol. 2008, 76, 15901611.

2. Ferrari, E.; Pignedoli, F.; Imbriano, C.; Marverti, G.; Basile, V.; Venturi, E.; et al. J. Med. Chem. 2011, 54, 8066-8077.

3. Weber, W.M.; Hunsaker, L.A.; Abcouwer, S.F.; Deck, L.M.; Jagt, D.L.V. Bioorg. Med. Chem. 2005, 13, 3811-3820.

4. Kim, H.K.; Yang, C.H. Bull. Korean Chem. Soc. 2004, 25, 1769-1774.

5. Nurfina, A.N.; Reksohadiprodjo, M.S.; Timmerman, H.; Jenie, U.A.; Sugiyanto, D.; van der Goot, H. Eur. J. Med. Chem. 1997, 32, 321-328.

6. Pabon, H.J.J. Rec. Traw. Chim. 1964, 83, 379386.

7. Shao, W.Y.; Cao, Y.N.; Yu, Z.W.; Pan, W.J.; Qiu, X.; Bu, X.Z.; An, L.K.; Huang, Z.S.;Gu,L. Q.; Chan, L.S.C.Tetrahedron Lett. 2006, 47, 4085-4089.

8. Leonard, J.; Lygo, B.; Procter, G. Advanced Practical Organic Chemistry 2nd edn. Blackie Academic and Professional, Glasgow (1995), 100-101.

9. Kofron, W.G.; Baclawski, L.M.J. Org. Chem.1976, 41, 1879-1880.
10. Fitton, A.O.; Smalley, R.K. Practical Heterocyclic Chemistry. Academic press; London (1968), 28.

11. Changtam, C.; Hongmanee, P.; Suksamrarn, A. Eur. J. Med. Chem. 2010, 45, 4446-4457.

12. Micetich, R.G. Can. J. Chem. 1970, 48, 20062014.

13. Kashima, C.; Tobe, S.; Sugiyama, N.; Yamamoto, M. Bull. Chem. Soc. Japan 1973, 46, 310-313.

14. Brunelle, D.J. Tetrahedron Lett. 1981, 22, 3699-3702.

15. Cermak, J., Nguyen Thi, T.H.;Veelak Krupkova, A. Molecules 2011, 16, 4031-4044.

16. Baraldi, P.G.; Barco, A.; Benetti, S.; Moroder, F.; Pollini, P.G.; Simoni, D. J. Org. Chem. 1983,48, 1297-1302.

17. Akhrem, A.A., Lakhvich, F.A., Khripach, V.A.; Klebanovich, I.B. Tetrahedron Lett. 1976, 17, 3983-3984.

18. Nitta, M.; Kobayashi, T. J. Chem. Soc., Chem. Commun.1982, 20, 1401-1406.

19. Kenar, J. A. J. Am. Oil. Chem. Soc. 2003, 80, 1027-1032.

20. Akhrem, A.A.; Lakhvich, F.A.; Khripach, V.A., Klebanovich, I.B. Chem. Heterocycl. Compd. 1979, 15, 194-198. 